\title{
BMJ Global Health Access to safe blood in low-income and middle-income countries: lessons from India
}

\author{
Hillary E Jenny, ${ }^{1,2,3}$ Saurabh Saluja, ${ }^{2,3,4}$ Rachita Sood, ${ }^{2,3,5}$ Nakul Raykar, ${ }^{2,3,6}$ \\ Raman Kataria, ${ }^{7}$ Ravindranath Tongaonkar, ${ }^{8}$ Nobhojit Roy ${ }^{9}$
}

To cite: Jenny HE, Saluja S, Sood R, et al. Access to safe blood in low-income and middle-income countries: lessons from India. BMJ Glob Health 2017;2:e000167. doi:10.1136/ bmjgh-2016-000167

Received 24 August 2016 Revised 13 February 2017 Accepted 16 February 2017 Published Online First

Published Online First 11 May 2017

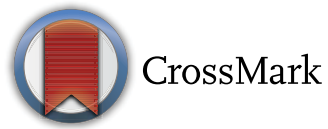

For numbered affiliations see end of article.

Correspondence to Dr Hillary E Jenny; hillary.jenny@icahn.mssm.edu

\section{ABSTRACT}

Timely, affordable access to screened blood is essential to the provision of safe surgical care and depends on three key aspects: adequate volume of blood supply, safe protocols for blood donation and transfusion, and appropriate regulation to ensure safe, equitable and sustainable distribution. Many low-income and middle-income countries experience a deficit in these categories, particularly in rural areas. We draw on the experience of rural surgical practitioners in India and summarise the existing literature to evaluate India's blood banking system and discuss its major barriers to the safe and equitable provision of blood. Many low-income and middle-income countries struggle with accruing a sufficient voluntary, unpaid blood donation base to meet the need. Efforts to increase blood supply through mandatory family replacement donations can lead to dangerous delays in care provision. Additionally, prohibition of unbanked, directed blood transfusion restricts the options of health practitioners, particularly in rural areas. Blood safety is also a significant concern, and efforts must be taken to decrease the risk of transfusion-transmitted infections and inform and treat donors who test positive. Lastly, blood banking systems need a centralised governing body to ensure fair prices for blood, promote comprehensive transfusion reporting and increase system-wide transparency and accountability.

\section{INTRODUCTION}

Timely, affordable access to screened blood is essential to the provision of safe surgical care. This depends on three key aspects: adequate volume of blood supply, safe protocols for blood donation and transfusion, and appropriate regulation to ensure safe, equitable and sustainable distribution. Many low-income and middle-income countries (LMICs) experience a deficit in these categories, particularly in rural areas.

We draw on the experience of rural surgical practitioners in India and on the existing literature to evaluate India's blood banking system and discuss its major barriers to the safe and equitable provision of blood. Specifically, our findings are informed by a series of discussions between the writing team and approximately 50 rural surgery practitioners

\section{Key questions}

What is already known about this topic?

- Blood banking is challenging in low-income and middle-income countries.

- Blood products are not always available in high enough quantity and at the time they are needed in low-resource settings.

- Problems such as transfusion-transmitted infections are a significant issue in these settings.

\section{What are the new findings?}

- This paper synthesises the available literature, combined with input from rural surgical practitioners in India, to identify key facets of any blood banking system: adequate volume of blood supply, safe protocols for blood donation and transfusion, and appropriate regulation.

- We identify key challenges to providing safe, timely access to blood and blood products in India, including the prohibition of unbanked directed blood transfusion (UDBT).

- Suggestions to overcome these challenges in India are proposed, such as implementing more centralised blood banking regulation and legalising UDBT. These solutions may be applicable to other low-income and middle-income countries with similar problems.

\section{Recommendations for policy}

- India would benefit from increased transparency in their blood banking system. This could be achieved through mandatory centralised reporting of the source, and the amount and type of blood donated to blood banks. Adding blood and blood products to India's National List of Essential Medicines will also improve pricing transparency and affordability.

- Extend the services of blood banks and storage centres to smaller towns and rural areas by removing the requirement for family replacement donation, decreasing the current blood bank statutory requirements and legalising regulated UDBT.

- These recommendations may be helpful in other low-income and middle-income countries, as many face blood banking challenges similar to India's. 
during telephone focus groups and a global surgery symposium held at the Association of Rural Surgeons of India's (ARSI) 2015 annual meeting in Karad, India. We place these discussions into context through studying the pre-existing literature by the WHO, medical literature in peer-reviewed journals and news publications. To improve access to safe, timely blood in India, we propose specific policy changes as well research studies to enhance the current body of literature. As our findings are placed in the context of LMICs as a group, these recommendations may have implications for addressing challenges faced across LMICs.

\section{VOLUME}

A volume of banked blood adequate for the population is a basic requirement for safe surgical care. The Lancet Commission on Global Surgery recommends at least 15 units/1000 people/year. ${ }^{1}$ According to 2015 WHO data, the median blood donation rate in high-income countries (HICs) is 36.8 units/1000 people/year, compared with 11.7 in middle-income countries and 3.9 in low-income countries (LICs). ${ }^{2}$ In India, a country with a population of $1.2 \mathrm{billion}$, about four units of blood are donated per 1000 people/year. ${ }^{3}$ Despite the inadequate blood volume in LMICs, little research has been done to further characterise this disparity and the consequences of not meeting these needs, particularly in rural areas.

A consistent, healthy volunteer donor base is essential to closing the gap in blood availability, but in many LMICs, blood donations come from a mix of voluntary non-remunerated donors (VNRD), paid donors and replacement donations, which occur when family members donate a unit of blood to replace each unit used by their friend or relative. While the WHO advocates for use of VNRD, paid donors are often used in LMICs. ${ }^{24}$ In fact, paid or family replacement donations account for more than $50 \%$ of the blood supply in 72 countries, 64 of which are LMICs. ${ }^{2}$

In India, the Supreme Court banned blood selling in 1998 , yet only $20 \%$ of the country's donations are not VNRD. ${ }^{15}$ Professional donors may pose as a relative or friend of the patient, which is difficult for blood banks to verify. ${ }^{6}$ Replacement donations are also a critical part of the current system. Allowing family replacement donations is, in LMICs, an efficient way to capitalise on willing donors to boost low supply volume. ${ }^{78}$ However, requiring a replacement donation may lead to delays in care-oftentimes the transfusion will not be administered to the patient in need until a family member travels to a blood bank, donates blood then returns. These delays in care can be fatal. ${ }^{9}$

Voluntary donation is further limited by chronic health conditions and cultural factors. Within India, overall deferral rates can be as high $11.6 \%$. Among these deferrals, anaemia is frequently reported as one of the leading factors, accounting for up to $77.9 \%$ of female deferrals and up to $37 \%$ of male deferrals. Sub-Saharan Africa has a similar problem, with overall deferral rates as high as
$16 \%$ and anaemia accounting for up to $73.6 \%$ of female and $38.5 \%$ of male deferrals. ${ }^{10-13}$ Several studies assessing knowledge, attitudes and practices of voluntary donors in various regions of India and Sub-Saharan Africa have also found that lack of awareness as well as misconceptions, such as blood donation leading to permanent weakness, infertility, accelerated ageing and fear of religious/ familial disapproval or exposure of their blood to witchcraft, are barriers to voluntary donation. ${ }^{14-18}$

India also experiences a significant rural-urban disparity in distribution of blood supply. Whereas $68 \%$ of the country's population is rural, most of its blood banks are located in urban areas. ${ }^{19}$ Blood storage centresfacilities where blood is not collected, but is acquired from central blood banks and stored-were introduced in 2001 to increase access in such geographically remote areas. However, blood storage centres are often insufficient, especially in emergency situations. ${ }^{520}$ Some rural surgeons also attest that in many public hospitals, which often serve as the first point of care for many patients, blood storage centres exist only in name. These centres are reportedly rarely operational due to multiple factors, including the central blood bank requirement for a family replacement donation, preferential deferral of nearly expired or unwanted blood group units to the storage centre, and the challenges inherent to the requirement of a constant and reliable power supply to operate the blood storage refrigerator and other necessary appliances. For example, one surgeon reported that the refrigerated vehicle meant to transport blood from the central blood bank to the storage centre has never left the blood bank. Another clinician reported that when he has attempted to retrieve blood from the blood storage centre nearest to his clinic, he was told they could not deliver blood as he was located $>40 \mathrm{~km}$ away, effectively eliminating his supply to blood products.

Many rural clinicians seek to address the issue of blood volume shortage through unbanked directed blood transfusion (UDBT). ${ }^{5} 921$ In UDBT, donors undergo rapid testing for transfusion-transmitted infections (TTIs), followed by phlebotomy and subsequent transfusion of the patient. In India, UDBT has been illegal in the civilian population since 1998 and remains so, despite promises by the Drug Technical Advisory Board to legalise the procedure in 2014. In March 2016, an amendment legalising UDBT gained support of the professional societies of India's surgeons (The Association of Surgeons of India; ASI), rural surgeons (ARSI), obstetricians and gynaecologists (Federation of Obstetrics and Gynaecological Societies of India; FOGSI), orthopaedists (Indian Orthopaedic Association; IOA) and anaesthesiologists (Indian Society of Anaesthesiologists; ISA). Moreover, the 2015 National Health Policy acknowledged that UDBT is the only feasible and safe option in some contexts (Ministry of Health and Family Welfare (MOHFW) 2014, Sect 4.3.4.3).

While UDBT is banned in civilian practice, it is permissible in the armed forces-a policy justified by the tight 
regulation and monitoring of the military's controlled environment. Some criticise UDBT, fearing increased rates of TTIs due to the use of rapid tests. However, in practice, banking is not always a significant improvement over UDBT. Although some studies have found rapid tests to be less sensitive, these rapid tests are frequently used by Indian blood banks due to resource limitations. ${ }^{22}{ }^{23}$ In addition, both India's National AIDS Control Organization (NACO) and the WHO have deemed rapid tests acceptable in remote locations or emergency situations. ${ }^{22} 24$ Another criticism is that UDBT involves transfusions of whole blood rather than components. However, India separates just $35 \%^{4}$ and LMICs on average $45 \%^{2}$ of banked blood into components, meaning that even banked blood is often transfused whole. In resource-limited environments, even HICs consider fresh whole blood transfusion acceptable in military and natural disaster settings. ${ }^{25}$ Lastly, studies have demonstrated no greater risk of morbidity or mortality with fresh versus banked blood ${ }^{26}$ Faced with these realities, proponents of UDBT argue that with appropriate regulation, UDBT may be an appropriate way to help address the unmet need for blood in the rural setting: guidelines can be set up to ensure safety and reporting, rapid tests with the highest possible sensitivity will be used, and accountability will lie clearly with the transfusing clinician. ${ }^{5}$

Given the significant financial capital associated with the blood banking system, some also question whether opposition to UDBT is driven by financial interests in which opponents are tied to the sale of blood and its products, or use their involvement with the blood banking system to gain political capital. ${ }^{27}$ Regardless of the reasons for its prohibition, without UDBT, many rural clinicians are left with few options to treat health emergencies requiring blood. ${ }^{1}$ This issue of a volume shortage compounded by geographical access challenges is not unique to India, but rather a concern for many LMICs who may also benefit from UDBT.

\section{SAFETY}

Even when blood is available, safety protocols for donation and transfusion are crucial to ensure that TTIs are not passed onto the donor or recipient. The WHO recommends all blood donations be screened for HIV, hepatitis $\mathrm{B}$ virus (HBV), hepatitis $\mathrm{C}$ virus (HCV) and syphilis, and recommends universal screening for Chagas disease, human $\mathrm{T}$ cell lymphotropic virus type I and II and cytomegalovirus in countries where these diseases are particularly prevalent. ${ }^{28}$ However, such extensive screening is not universal practice in LIC blood banks and often has little oversight: $34 \%$ of blood laboratories in LICs are subjected to external quality assessment monitoring compared with $81 \%$ in HICs, resulting in an average transfusion-transmitted HIV prevalence of $1.080 \%$ versus $0.003 \% .^{2}$ A consistent, voluntary donor base practising repeated donations (VNRDs), a concept more prevalent in HICs than LMICs, may also assist in driving down the prevalence of TTIs. ${ }^{2}$

India currently mandates testing for HIV, HBV, HCV, syphilis and malaria. Although prevalence of these infections and incidence of these TTIs in transfusion recipients varies, $\mathrm{HBV}$ can be as prevalent as affecting $24.3 \%$ of potential donors, ${ }^{29}$ with TTI-related HBV incidence rates as high as $3.59 \% .^{30}$ Many factors may contribute to TTI transmission, including failure of adequate screening. Type of TTI screening used varies based on resource availability-although the first-line screening test for HIV is ELISA, some blood banks resort to rapid diagnostic tests (RDTs) due to paucity of resources. ${ }^{22}{ }^{23}$ The sensitivity and specificity of RDTs in comparison with ELISA are still being researched-some studies have reported similar results, ${ }^{30}{ }^{31}$ while others have found RDTs to have inferior sensitivity compared with ELISA. ${ }^{23} 32$ However, both NACO and the WHO approve the use of rapid tests in emergency scenarios or in small laboratories with low testing volume. ${ }^{33}$

In addition to recipient safety, donor safety should not be overlooked. The National Blood Transfusion Council (NBTC) of India recommends informing blood donors when they screen positive for TTIs, and as of 2002 requires obtaining written consent for TTI testing at the time of donation. As many blood banks do not have the ability to perform confirmatory tests for TTIs, HIV-reactive donors are to be contacted and referred to Integrated Counselling and Testing Centres, and donors reactive for HBV or HCV are referred to a gastroenterologist for management. ${ }^{22}$ However, in practice, many banks do not make active attempts to inform donors and instead ask the donor to contact the blood bank, a task that as few as $20 \%$ of donors undertake. ${ }^{34}$ Therefore, those who are true-positives may never become aware of their status, and those with false-negatives may never receive further testing.

\section{REGULATION}

Finally, a major problem faced by blood banking systems in LMICs is the fragmentation of their organisation and regulation. Many countries have a decentralised model that heavily relies on volunteer organisations, private blood banks and non-governmental organisations (NGOs). Without oversight, blood banks often do not follow the national or state guidelines restricting the price of blood. For example, in India, the cost of blood has been restricted to 1450 rupees (US\$21-25) for non-governmental banks and 1050 rupees (US\$15-18) for governmental blood banks. However, many banks do not adhere to these requirements, charging unaffordable fees as high as 14500 rupees (US\$214-247) for single donor platelets, thereby impeding access to blood even when supply is sufficient. ${ }^{35-39}$ In addition, the unmet need for blood has created a black market, with surreptitious sales of units sourced from both willing paid donors and donors held captive in blood farms. ${ }^{38} 40$ Unstandardised or poorly overseen quality 
assurance measures can also lead to unstandardised laboratory and manufacturing practices. ${ }^{20}$ Lack of centralised regulation also interferes with comprehensive transfusion reporting and leads to a lack of transparency and accountability: it is often difficult to ascertain where blood is available and how it was obtained.

A national blood policy is essential in addressing these issues. This policy should establish a cohesive, centrally managed blood transfusions service (BTS) and provide a framework for quality management. ${ }^{12} 4142$ India has a national blood policy, which established the NBTC and State Blood Transfusions Councils as responsible for the national, government-controlled BTS. This BTS is coordinated by the NACO. However, this system is highly fragmented as the management of blood banks varies- the government manages $39.4 \%$, private hospitals $28.8 \%$, and the remaining $35.1 \%$ are managed by NGOs, the Indian Red Cross Society and private charities. ${ }^{4627}$ However, blood bank licensure and oversight of good manufacturing practices are the responsibilities of the Central Drugs Standard Control Organisation. ${ }^{43}$ In addition, there is currently no medium for centralised transfusion reporting that would enable evaluation of blood availability of blood across all sectors. This fragmented organisation leads to inadequate reporting and also fails to ensure the needs of all practitioners and their patients are represented: many of the legislative decisions about blood banking, including the prohibition of UDBT, are made in cities without the perspective of the rural health clinician or the rural patientseeking care.

\section{NEXT STEPS}

LMICs face significant challenges in ensuring adequate blood volume, transfusion safety and comprehensive regulation of blood and its products. Like LMICs around the world, India's annual blood supply does not meet the need. Rural practitioners' access is further precluded by geographical isolation from blood banks and non-functional blood storage centres. When banked blood is available, costs to patients are variable, exorbitant and often include the need for replacement donation by a family member. Practitioners attempting to meet the need for blood through UDBT face legal repercussions, even when the patient's life is in danger. Finally, a fragmented, non-transparent regulatory system is unable to manage these challenges to effectively meet rural surgeons' needs.

In India, we propose a call to action in academic transfusion medicine. This paper was limited both by the experiences of the practitioners informing our recommendations and by the paucity of medical literature in peer-reviewed journals. Therefore, we relied heavily on policy documents and grey literature in addition to the experiences of our rural surgeon colleagues. However, this limitation in turn identified an area of weakness in academic literature; the dearth of peer-reviewed articles on blood transfusion systems, the quantified need for blood and consequences of inadequate supply illustrate a strong need for more academic research in this field. These increased academic efforts may in turn facilitate effecting policy changes necessary to improve the state of blood banking in India.

We propose four targeted policy changes to address the issues prevalent in India's blood banking system. Although these are targeted to India, these ideas may be applied to other LMICs facing similar barriers. First, NACO and the NBTC must enhance transparency through mandatory centralised reporting of the source, amount and type of blood donated to blood banks. Such an initiative would promote blood banking transparency and responsible sourcing of blood, moving India towards the goal of $100 \%$ VNRD.

Second, it is imperative to extend the services of blood banks and storage centres to smaller towns and rural areas by both increasing functionality of blood storage centres and decreasing the current blood bank statutory requirements. To achieve this end, blood storage centres should be made fully functional, without the handicap of a replacement unit requirement. In addition, blood statutory requirements, such as the mandatory employment of a pathologist and transfusion medicine specialist in all blood banks, must be relaxed. Although these requirements intend to ensure safety for both donor and recipient, they effectively function as barriers to blood transfusion access in rural areas.

Third, as blood and blood products are already on the WHO Model List of Essential Medicines, ${ }^{44}$ they must be added to India's National List of Essential Medicines to improve affordability. Finally, carefully regulated UDBT should be made legal in select circumstances, along with an amendment to the Rules in Drug and Cosmetics Act to ensure that the Food and Drug Administration can appropriately regulate this practice. Legalising regulated UDBT will help ensure that when patients urgently need blood, their lives are not further endangered by inadequate blood supply or unsafe transfusion practices. In these emergent cases, UDBT was declared 'the only feasible and safe option' in India's National Health Policy 2015 draft, and should be legally supported in that context. ${ }^{45}$

Insufficient VNRD-sourced blood volume, transfusion safety concerns, and opaque, decentralised regulatory bodies were personally observed as problems by contributors in India. However, we believe these challenges may be experienced by other LMICs. Insights from our proposed solutions for India may be applied to address the needs of LMICs across the world.

\section{Author affiliations}

${ }^{1}$ Department of Medical Education, Icahn School of Medicine at Mount Sinai, New York, USA

${ }^{2}$ Program in Global Surgery and Social Change, Harvard Medical School, Boston, USA

${ }^{3}$ Department of Plastic and Oral Surgery, Boston Children's Hospital, Boston, USA ${ }^{4}$ Department of Surgery, Weill Cornell Medicine, New York, USA

${ }^{5}$ Department of Medical Education, University of Miami Miller School of Medicine, Miami, FL, USA 
${ }^{6}$ Department of Surgery, Beth Israel Deaconess Medical Center, Boston, MA, USA ${ }^{7}$ Department of Surgery, Jan Swasthya Sahyog, Beltookri, Chhattisgarh, India ${ }^{8}$ Association of Rural Surgeons of India, Chennai, Tamil Nadu, India ${ }^{9}$ Department of Surgery, BARC Hospital, Mumbai, Maharashtra, India

Contributors HEJ, primary writer, data collector and interpreter; SS, writer, data collector and interpreter; RS, writer, data collector and interpreter; NR, writer, reviewer; RK, provider of primary data, writer, reviewer; RT, provider of primary data, writer, reviewer; NR, provider of primary data, reviewer.

Competing interests All authors have completed the unified competing interest form and declare no support from any organisation for the submitted work, financial relationships with any organisations that might have an interest in the submitted in work in the previous 3 years, and no other relationships or activities that could appear to have influenced the submitted work.

Provenance and peer review Not commissioned; externally peer reviewed.

Data sharing statement № additional data are available.

Open Access This is an Open Access article distributed in accordance with the Creative Commons Attribution Non Commercial (CC BY-NC 4.0) license, which permits others to distribute, remix, adapt, build upon this work non-commercially, and license their derivative works on different terms, provided the original work is properly cited and the use is non-commercial. See: http://creativecommons.org/ licenses/by-nc/4.0/

(c) Article author(s) (or their employer(s) unless otherwise stated in the text of the article) 2017. All rights reserved. No commercial use is permitted unless otherwise expressly granted.

\section{REFERENCES}

1. Meara JG, Leather AJ, Hagander L, et al. Global Surgery 2030: evidence and solutions for achieving health, welfare, and economic development. Int J Obstet Anesth 2016;25:75-8.

2. World Health Organization. Blood safety and availability. (Fact sheet \#279). [Internet] July 2016. 1/12/2016 http://www.who.int/ mediacentre/factsheets/fs279/en/.

3. Kralievits K, Raykar N, Shrime M, et al. The lancet comission on global surgery appendix 2.6: blood: an estimation of optimal blood donation rate and a review of the blood supply literature. The Lancet 2015:51-76.

4. Choudhury N. Blood transfusion in borderless South Asia. Asian J Transfus Sci 2011;5:117-20.

5. Bhaumik S. Unbanked direct blood transfusions should be legal, say Indian surgeons. BMJ 2013;347:f5623.

6. Nair SC, Mammen JJ. Repeat voluntary non-remunerated blood donor is the best quality indicator for blood safety. Indian $J$ Med Res 2015;141:749-52.

7. Allain JP. Moving on from voluntary non-remunerated donors: who is the best blood donor? Br J Haematol 2011;154:763-9.

8. Allain JP, Sibinga CT. Family donors are critical and legitimate in developing countries. Asian J Transfus Sci 2016:10:5-11.

9. Kataria R, Jain $Y$. A lifeline that rural India cannot do without. The Hindu 2013 http://www.thehindu.com/opinion/op-ed/a-lifeline-thatrural-india-cannot-do-without/article5164781.ece.

10. Agnihotri N. Whole blood donor deferral analysis at a center in Western India. Asian J Transfus Sci 2010;4:116-22.

11. Ekwere TA, Ino-Ekanem M, Motilewa OO, et al. Pattern of blood donor deferral in a tertiary hospital, South-south, Nigeria: A three-year study review. Int JBlood Transfus Immunohematol 2014;4:7-13.

12. Kouao MD, Dembelé B, N'Goran LK, et al. Reasons for blood donation deferral in sub-Saharan Africa: experience in Ivory Coast. Transfusion 2012;52:1602-6.

13. Sundar P, Sangeetha SK, Seema DM, et al. Pre-donation deferral of blood donors in South Indian set-up: An analysis. Asian J Transfus Sci 2010;4:112-5.

14. Dubey A, Sonker A, Chaurasia R, et al. Knowledge, attitude and beliefs of people in North India regarding blood donation. Blood Transfus 2014;12:s21-7.

15. Kowsalya V, Vijayakumar R, Chidambaram R, et al. A study on knowledge, attitude and practice regarding voluntary blood donation among medical students in Puducherry, India. Pak J Biol Sci 2013:16:439-42.

16. Agrawal A, Tiwari AK, Ahuja A, et al. Knowledge, attitude and practices of people towards voluntary blood donation in Uttarakhand. Asian J Transfus Sci 2013;7:59-62.
17. Harrington AH. Blood banks in Kumasi, Ghana: Social barriers preventing volunteer blood donations. University of Michigan Undergraduate Research Journal 2012;9:4-7.

18. Umeora OU, Onuh SO, Umeora MC. Socio-cultural barriers to voluntary blood donation for obstetric use in a rural Nigerian village. Afr J Reprod Health 2005;9:72-6.

19. Rural population. 2014. http://data.worldbank.org/indicator/SP.RUR TOTL.ZS.

20. Ramani KV, Mavalankar DV, Govil D. Study of blood-transfusion services in Maharashtra and Gujarat States, India. J Health Popul Nutr 2009;27:259-70.

21. Pandey K. Bloodless Ban: Health activists demand removal of ban on unbanked direct blood transfusion. Down To Earth 2016;1 http:// www.downtoearth.org.in/coverage/bloodless-ban-42568.

22. Chaurasia R, Zaman S, Das B, et al. Screening donated blood for transfusion transmitted infections by serology along with NAT and response rate to notification of reactive results: An Indian Experience. J Blood Transfus 2014:2014:1-6.

23. Mehra B, Bhattar S, Bhalla P, et al. Rapid tests versus ELISA for screening of HIV infection: our experience from a voluntary counselling and testing facility of a tertiary care centre in North India. Isrn Aids 2014;2014:1-5.

24. Bharucha ZS, Jolly JG, Ghosh K, et al. Standards for Blood Banks \& Blood Transfusion Services. National AIDS Control Organisation $\mathrm{MoHaFW}$, Government of India. editor. New Delhi: National AIDS Control Organisation, 2007:p. 35.

25. Goforth CW, Tranberg JW, Boyer P, et al. Fresh Whole Blood Transfusion: Military and Civilian Implications. Crit Care Nurse 2016;36:50-7.

26. Alexander PE, Barty R, Fei $\mathrm{Y}$, et al. Transfusion of fresher vs older red blood cells in hospitalized patients: a systematic review and metaanalysis. Blood 2016;127:400-10.

27. Choudhury N. Management in Indian blood banking system: true reality. Asian J Transfus Sci 2009;3:57-9.

28. World Health Organization. Screening Donated Blood for Transfusion-Transmissible Infections: Recommendations 2009 https://www.ncbi.nlm.nih.gov/books/NBK142990/.

29. Choudhury N, Phadke S. Transfusion transmitted diseases. Indian J Pediatr 2001:68:951-8.

30. Kannangai R, Ramalingam S, Selvaraj PK, et al. Hospital-based evaluation of two rapid human immunodeficiency virus antibody screening tests. J Clin Microbiol 2000;38:3445-7.

31. Lien TX, Tien NT, Chanpong GF, et al. Evaluation of rapid diagnostic tests for the detection of human immunodeficiency virus types 1 and 2, hepatitis B surface antigen, and syphilis in Ho Chi Minh City, Vietnam. Am J Trop Med Hyg 2000;62:301-9.

32. Torane VP, Shastri JS. Comparison of ELISA and rapid screening tests for the diagnosis of HIV, Hepatitis B and Hepatitis C among healthy blood donors in a tertiary care hospital in Mumbai. Indian $J$ Med Microbio/ 2008;26:284-5.

33. World Health Organization. In vitro diagnostics and laboratory technology: Simple/Rapid tests. http://www.who.int/diagnostics_ laboratory/faq/simple_rapid_tests/en/.

34. Choudhury LP, Tetali $\mathrm{S}$. Ethical challenges in voluntary blood donation in Kerala, India. J Med Ethics 2007;33:140-2.

35. Roy SD, Tembhekari C. Govt lowers blood bag price by Rs 200 . The Times of India http://timesofindia.indiatimes.com/city/mumbai/Govtlowers-blood-bag-price-by-Rs-200/articleshow/45635553.cms.

36. Royl SD 1 in 3 Mumbai blood banks bleeds its customers: FDA The Times of India http://timesofindia.indiatimes.com/city/mumbai/ 1-in-3-Mumbai-blood-banks-bleeds-its-customers-FDA/articleshow/ 45313567.cms.

37. Chakraborty R. Mumbai: This is how BMC blood banks are cheating you. Mid-day 2016 http://www.mid-day.com/articles/bmc-bloodbanks-mumbai-news-mumbai-cost-of-blood-civic-hospitals/ 17654170.

38. Carney S. The Red Market: On the Trail of the World's Organ Brokers, Bone Thieves, Blood Farmers and Child Traffickers. 1 ed: William Morrow, 2011:150-93

39. Choudhury N. Prices of blood units in South East Asia. Asian Transfus Sci 2008;2:45-6.

40. Anand A. Blood for sale: India's illegal 'red market'. BBC News 2015 http://www.who.int/bloodsafety/StrategicPlan20082015AccessSafeBloodTransfusion.pdf.

41. World Health Organization. Universal access to safe blood transfusion. $2008 \mathrm{http}: / / w w w . w h o . i n t / b l o o d s a f e t y /$ StrategicPlan2008-2015AccessSafeBloodTransfusion.pdf.

42. World Health Organization. Consensus statement on good policy process for blood safety and availability. 2004 http://www.who.int/ bloodsafety/gcbs/Consensus_Statement_Forum_for_Good_Policy_ Process-04.pdf. 
43. Central Drugs Standard Control Organization. Blood Bank: Director General of Health Services, Ministry of Health and Family Welfare, Government of India;. 2014 http://cdsco.nic.in/forms/list.aspx?lid= $1813 \& l d=1$.
44. World Health Organization. 19th WHO Model List of Essential Medicines. 2015:26-7.

45. Ministry of Health \& Family Welfare. National Health Policy 2015 Draft. 2015 http://www.nhp.gov.in/sites/default/files/pdf/draft national_health_policy_2015.pdf. 